\title{
Dual-Band Butler Matrix for WLAN Systems
}

\author{
Carlos Collado*, Alfred Grau ${ }^{\dagger}$ and Franco De Flaviis ${ }^{\dagger}$
}

\begin{abstract}
*Technical University of Catalonia, Campus Nord D4-009, Barcelona, 08034, Spain (collado@tsc.upc.es) and ${ }^{\dagger}$ University of California at Irvine, Irvine, California 92697, USA (franco@uci.edu)
\end{abstract}

\begin{abstract}
This paper shows the design of a dual-band Butler matrix aimed to cover the bands corresponding to the standards IEEE802.11a/b, with particular interest in WLAN systems, at the 2.4 GHz and $5 \mathrm{GHz}$ bands. Measurements of the individual dualband components which compose the Butler matrix are presented. Those components are: quadrature hybrid and $0 \mathrm{~dB}$ coupler. The phase shifter design is also described. A final design of the Butler matrix is proposed and measured. Over the proposed frequency range, the Butler matrix exhibits phase errors and couplings of within $13.5^{\circ}$ and $-6.7 \pm 0.8 \mathbf{d B}$, respectively. A performance prediction of the Butler matrix connected to an array of isotropic and perfectly matched antennas separated half-wavelength in air, is also briefly commented.
\end{abstract}

\section{INTRODUCTION}

Butler matrices are used to design multi-port amplifier [1][2], and especially to design smart antennas with switched radiation beams [3][4] for communications systems. Some of them are dual-band systems. Circuits working at both bands are useful to increase the integration level.

At microwave frequencies is difficult to design a circuit with the same behavior at two uncorrelated frequencies, so the above commented systems often share only a dual-band antenna and the frequency bands are separated using diplexer.

The elements of a Butler matrix are quadrature hybrids, a $0 \mathrm{~dB}$ coupler and phase shifters. There are two possible approaches for the design of those circuits for its use in a dual-band system: to use broadband elements or dual-band elements. The dual-band approach has been followed in this case.

The design of the dual-band Butler matrix is aimed to be use in WLAN systems and cover the frequencies corresponding to the standards IEEE802.11b at $f_{1}=2.45 \mathrm{GHz}$, and IEEE802.11a at $f_{2}=5.25 \mathrm{GHz}$ and $f_{3}=5.8 \mathrm{GHz}$.

The proposed microstrip Butler matrix provides enhanced bandwidth for the upper frequency band at $5 \mathrm{GHz}$ in order to cover all its frequency range. Under our knowledge this is the first dual all planar Butler Matrix (BM) reported in the literature.

\section{Elements of the Butler Matrix}

An NxN Butler matrix can be used to feed an array of $\mathrm{N}$ antennas [5]. The scheme of a $4 \times 4 \mathrm{BM}$ is shown in Fig. 1. From the transmission point of view, the BM distributes the incoming signal at one TX/RX port to the ports in the array of antennas. If conveniently designed, the signals at the four ANT-ports have constant differential phase shift between consecutive radiators. The superposition of all the phased beams results in a more directional radiation beam pointing to a certain direction. The shape of the radiation pattern and

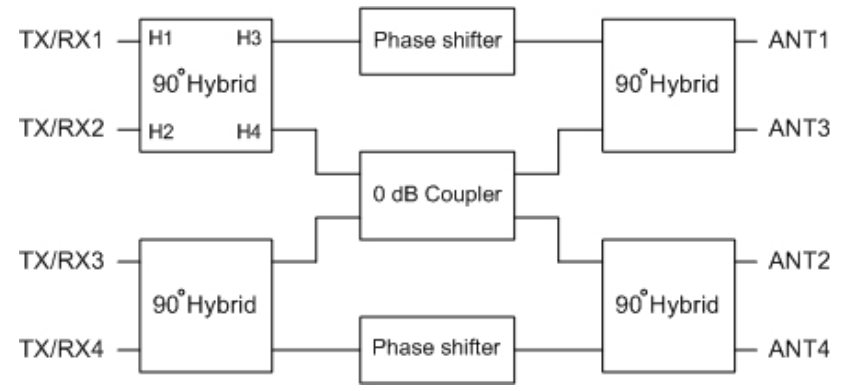

Fig. 1. Schematic of the proposed dual-band Butler matrix

TABLE I

DIFFERENCES OF PHASES BETWEEN CONSECUTIVE ANTENNAS

\begin{tabular}{|c|c||c|c|c|c|}
\hline $\begin{array}{c}\text { Hybrid } \\
\text { phase } \\
\left(\Delta \varphi_{h}\right)\end{array}$ & $\begin{array}{c}\text { Shift } \\
\text { phase } \\
(\Delta \phi)\end{array}$ & $\mathrm{TX} / \mathrm{RX} 1$ & $\mathrm{TX} / \mathrm{RX} 2$ & $\mathrm{TX} / \mathrm{RX} 3$ & $\mathrm{TX} / \mathrm{RX} 4$ \\
\hline \hline $90^{\circ}$ & $-45^{\circ}$ & $45^{\circ}$ & $-135^{\circ}$ & $135^{\circ}$ & $-45^{\circ}$ \\
\hline $90^{\circ}$ & $135^{\circ}$ & $-135^{\circ}$ & $45^{\circ}$ & $-45^{\circ}$ & $135^{\circ}$ \\
\hline$-90^{\circ}$ & $45^{\circ}$ & $-45^{\circ}$ & $135^{\circ}$ & $-135^{\circ}$ & $45^{\circ}$ \\
\hline$-90^{\circ}$ & $-135^{\circ}$ & $135^{\circ}$ & $-45^{\circ}$ & $45^{\circ}$ & $-135^{\circ}$ \\
\hline
\end{tabular}

the direction where it points depends on which TX/RX port is used, so it is possible to switch between 4 beams, which moreover are orthogonal and uncorrelated.

As shown in Fig. 1 a BM consists of quadrature hybrids, cross-overs and phase shifters which provide adequate phase shifts to operate appropriately. Following the numeration of Fig. 1, if the differential phase $\Delta \varphi_{h}=\varphi_{h\left(S_{31}\right)}-\varphi_{h\left(S_{41}\right)}$ (where $\varphi_{h\left(S_{31}\right)}$ and $\varphi_{h\left(S_{41}\right)}$ are the phases of the $S_{31}$ and $S_{41}$ parameters respectively) of each hybrid is $\Delta \varphi_{h}=-90^{\circ}$, then the phase shifter has to change the phase of the incoming signal by $\Delta \phi=45^{\circ}$. On the other hand, if those differential phase shifts were to be $\Delta \varphi_{h}=90^{\circ}$, the amount to shift in that case would be $\Delta \phi=-45^{\circ}$.

However these two solutions are not the unique possibilities. Table I shows the differences of phases between consecutive antennas of all four possible combinations or designs, for each $\mathrm{TX} / \mathrm{RX}$ ports of the BM.

It is obvious that the performance of the overall system is the same for each solution, however in order to design a dualband butler matrix, we can take advantage of this flexibility by choosing the best suitable solution for each frequency, once one is concerned about the design of each individual component, and in particular during the design of the phase shifter commented later in section II-C.

We have tested individually the components: hybrid, $0 \mathrm{~dB}$ coupler and phase shifter, which were later combined together 


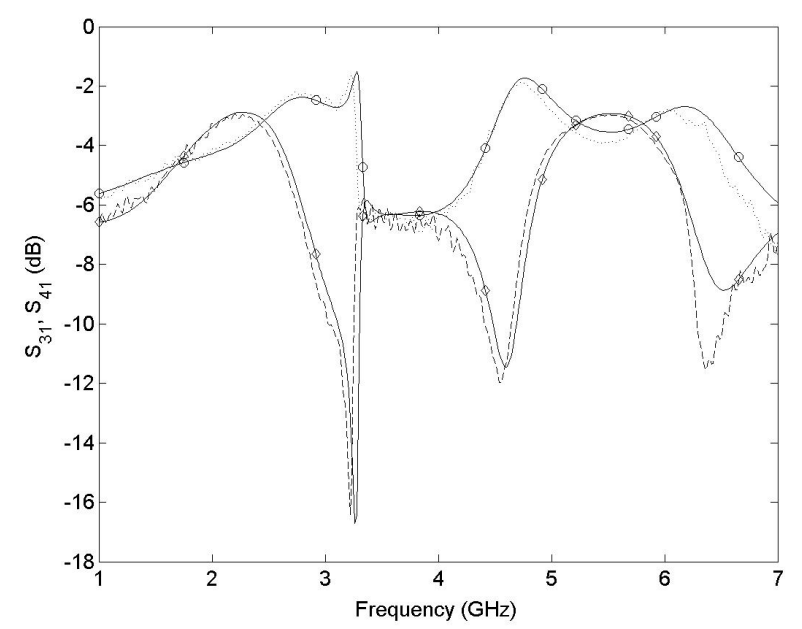

Fig. 2. Simulation (solid lines) and measurements (non-solid lines) of the insertion loss for the H1-H3 path, $S_{31}$ (circles line), and the H1-H4 path, $S_{41}$ (diamonds line).

TABLE II

MEASUREMENT DATA AT THE CORRESPONDING FREQUENCIES FOR THE US WLAN SERVICES

\begin{tabular}{|c||c|c|c|c|c|}
\hline Frequency & $\begin{array}{c}S_{31} \\
(\mathrm{~dB})\end{array}$ & $\begin{array}{c}S_{41} \\
(\mathrm{~dB})\end{array}$ & $\Delta \varphi_{h}\left(^{\circ}\right)$ & $\begin{array}{c}\text { Isolation } \\
(\mathrm{dB})\end{array}$ & $\begin{array}{c}\text { Return } \\
\text { Loss }(\mathrm{dB})\end{array}$ \\
\hline \hline$f_{1}=2.45 \mathrm{GHz}$ & -3.1 & -3.4 & -89.7 & -30 & -28 \\
\hline$f_{2}=5.25 \mathrm{GHz}$ & -3.6 & -3.2 & 91.1 & -27 & -27 \\
\hline$f_{3}=5.8 \mathrm{GHz}$ & -3.3 & -3.5 & 91.5 & -24 & -24 \\
\hline
\end{tabular}

to design the butler matrix of Fig. 7. The used dielectric was RO3006 with relative dielectric constant 6.15 , thickness 0.635 $\mathrm{mm}$ and metallization thickness $0.017 \mathrm{~mm}$.

\section{A. Dual-band hybrid}

Planar dual-band hybrids can be designed using 3 branchline $3 \mathrm{~dB}$ couplers in which the length of the coupling lines in one pair of the parallel arms is twice longer $(\lambda / 2)$ than in the other $(\lambda / 4)[6]$. These couplers can be designed to operate at two uncorrelated frequencies and they have large bandwidths at each frequency band. Fig. 7 shows the prototype that is used for the BM design.

The hybrid was designed to provide operativity for both the IEEE 802.11b and IEEE802.11a standards, in the $2.4 \mathrm{GHz}$ and the $5 \mathrm{GHz}$ band respectively. This hybrid was optimized to enhance the bandwidth of the upper frequency band in order to deal with the services allocated between $5.15 \mathrm{GHz}$ and 5.85 $\mathrm{GHz}$, in both the US and EU.

Figures 2, 3 and 4 shows the good agreement between simulation and measurements of the $\mathrm{S}$ parameters, and table II resumes the more relevant data at the corresponding frequencies for the US WLAN services.

\section{B. Dual-band 0 dB coupler}

A planar cross-over can be made by cascading two quadrature hybrids [3]. The layout of the proposed cross-over can be seen in Fig. 7. It was designed by cascading two dual hybrids like that described in the previous section and it is the

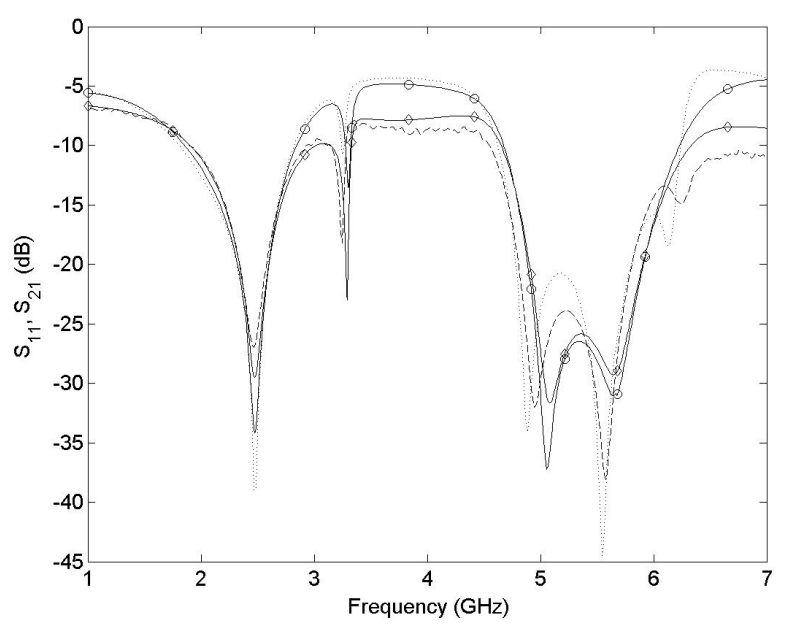

Fig. 3. Simulation (solid lines) and measurements (non-solid lines) of the return loss, $S_{11}$ (circles line) and isolation, $S_{21}$ (diamonds line).

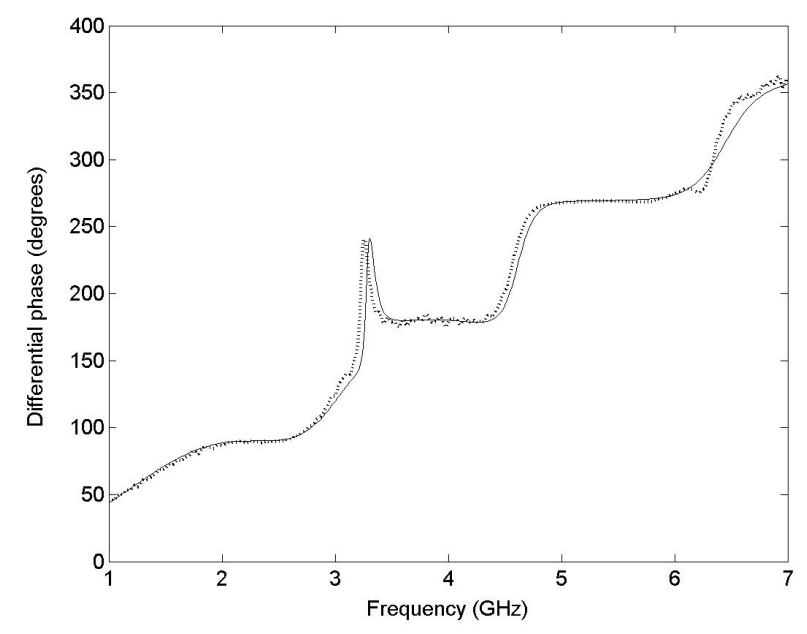

Fig. 4. Simulation (solid line) and measurements (doted line) of the phase difference between port $\mathrm{H} 3$ and $\mathrm{H} 4$.

result of an optimization process using the method of moments simulator (Momentum) from [7].

Figures 5 and 6 shows the simulations and measurements data of some of the main $\mathrm{S}$ parameters. The measured insertion losses are smaller than $0.7 \mathrm{~dB}$ for the three frequencies $(2.45$ $\mathrm{GHz}, 5.25 \mathrm{GHz}$ and $5.8 \mathrm{GHz}$ ) and the return losses and isolations better than $20 \mathrm{~dB}$.

\section{Phase shifter}

The phase shifter has to delay the signals an amount equal to the delay of the crossover, $\phi_{c}$, plus the needed phase shift $\Delta \phi$. That is, $\phi_{p}=\phi_{c}+\Delta \phi$ being $\phi_{p}$ the phase shifter phase delay. That means that the simulated phases $\phi_{c}$ of the crossover at each frequency, which are $47^{\circ}, 64^{\circ}$ and $-28^{\circ}$ at $2.45 \mathrm{GHz}$, $5.25 \mathrm{GHz}$ and $5.8 \mathrm{GHz}$ respectively, have to be added to one of those suggested in the second column of Table I taking into account that the designed hybrid has a $90^{\circ}$ differential phase shift between $\mathrm{H} 3$ port and $\mathrm{H} 4$ port (see Fig. 7) at $2.45 \mathrm{GHz}$, and 
TABLE III

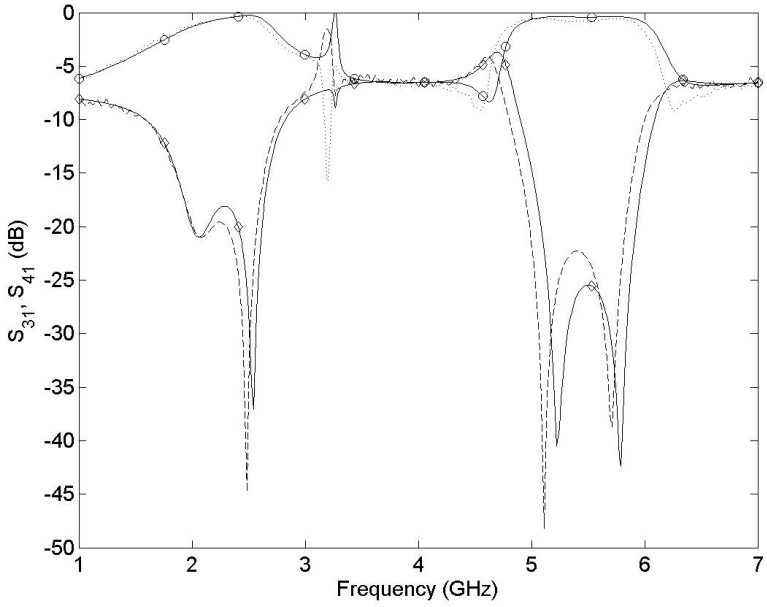

Fig. 5. Simulation (solid lines) and measurements (non-solid lines) of the insertion loss for the C1-C3 path, $S_{31}$ (circles line), and the C1-C4 path, $S_{41}$ (diamonds line).

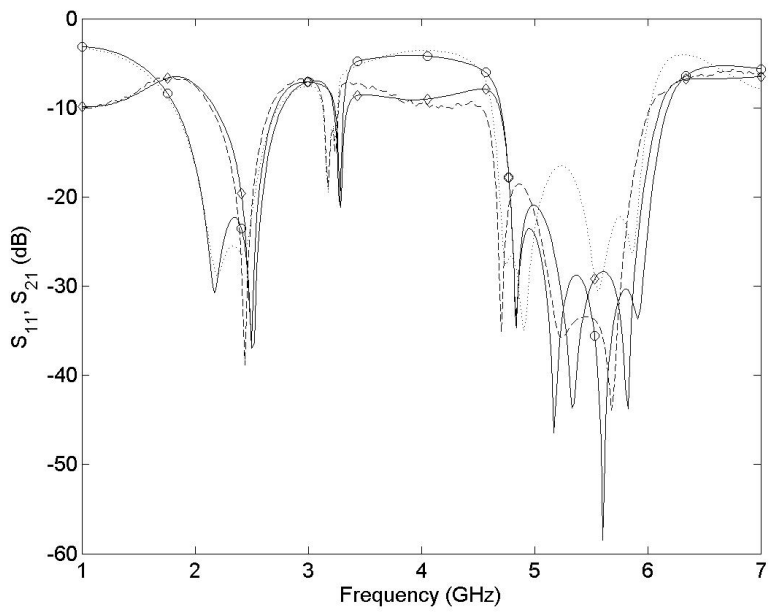

Fig. 6. Simulation (solid lines) and measurements (non-solid lines) of the return loss, $S_{11}$ (circles line) and isolation, $S_{21}$ (diamonds line).

$-90^{\circ}$ from $5.25 \mathrm{GHz}$ to $5.8 \mathrm{GHz}$. Table III shows a summary of the suitable phase shifts amounts for each component of the BM for two solutions of a total of four, at the three design frequencies. Those four possible combinations are the ones commented previously in table I.

A 50 Ohms transmission line of length $60.2 \mathrm{~mm}$ produce similar phase shifts that those values typed with bold style at Table III, therefore we chosen those as goals for the design of a more accurate phase shifter. The final design is a transmission line with specifically located and shaped meandered curves and it can be seen at Fig. 7. Such circuit produces the required phase shifts within a margin of error smaller than $2^{\circ}$, has insertion losses smaller than $0.6 \mathrm{~dB}$ and return losses better than $20 \mathrm{~dB}$.

\section{BUTLER MATRIX LAYOUT AND MEASUREMENTS}

The layout of the whole Butler matrix is shown in Fig. 7. The total area is $60 \times 60 \mathrm{~mm}$. In this version, no efforts to reduce
PHASE SHIFTS AT THE THREE DESIGN FREQUENCIES

\begin{tabular}{|c||c|c|c|}
\hline & $f_{1}$ & $f_{2}$ & $f_{3}$ \\
\hline \hline Hybrid $\left(\Delta \varphi_{h}\right)$ & $90^{\circ}$ & $-90^{\circ}$ & $-90^{\circ}$ \\
\hline Crossover $\left(\phi_{c}\right)$ & $47^{\circ}$ & $64^{\circ}$ & $-28^{\circ}$ \\
\hline $\begin{array}{c}\text { Phase shifter }\left(\phi_{p}\right) \\
\text { Solution } 1:\left(\Delta \phi=-45^{\circ}\right.\end{array}$ & & & \\
at $f_{1}$ and $\Delta \phi=45^{\circ}$ at $\left.f_{2,3}\right)$ & $\mathbf{2}^{\circ}$ & $109^{\circ}$ & $17^{\circ}$ \\
\hline $\begin{array}{c}\text { Phase shifter }\left(\phi_{p}\right) \\
\text { Solution } 2:\left(\Delta \phi=135^{\circ}\right.\end{array}$ & & & \\
at $f_{1}$ and $\Delta \phi=-135^{\circ}$ at $\left.f_{2,3}\right)$ & $182^{\circ}$ & $-\mathbf{7 1}^{\circ}$ & $-\mathbf{1 6 3}^{\circ}$ \\
\hline
\end{tabular}

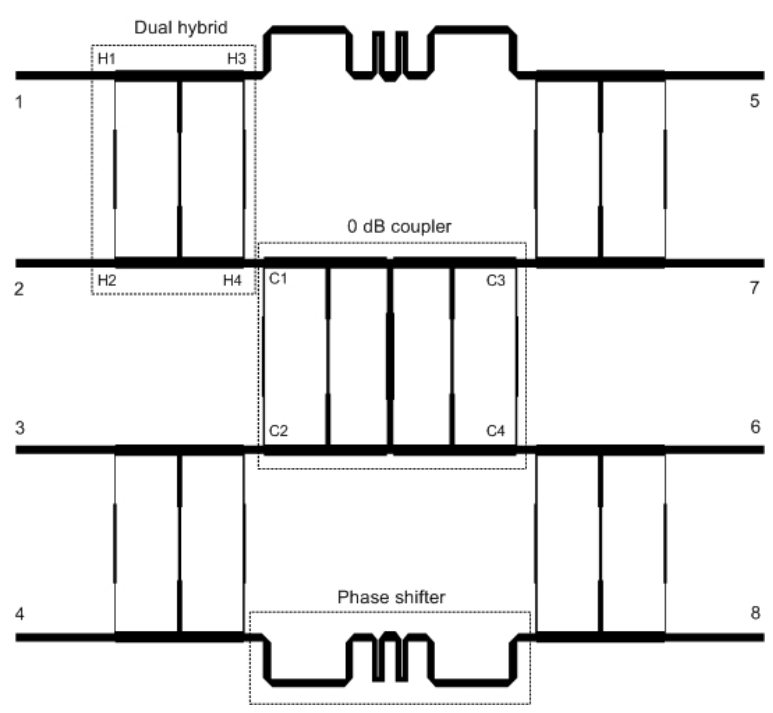

Fig. 7. Layout of the dual-band Butler matrix.

its size have been made and smaller design could be achieved by replacing the straight transmission lines of the hybrids by meander lines [8] or loading its branches with open stubs [9] [10].

Figures 8 and 9 shows the insertion losses of the network when it is feed at the port number 1 and 2 respectively. Note the large bandwidth of the second band around $5.5 \mathrm{GHz}$, in both ports. Port 3 and port 4 gives the same results due to the network symmetry.

Table IV shows the insertion losses at the 3 frequencies of interest when the signal is input in port 1 and 2 .

And Table $\mathrm{V}$ shows the differential phase shift between antenna ports, when the BM is feed at port 1 and 2. Similarly, the phases generated when feed from port 3 and port 4 are the same but with opposite sign, due to the network symmetry.

\section{CONCLUSION}

A planar microstrip dual-band Butler matrix has been proposed. It was designed to be use in multiband $(2.45 \mathrm{GHz}, 5.25$ $\mathrm{GHz}$ and $5.8 \mathrm{GHz}$ ) operation services (WLAN systems). The

TABLE IV

INSERTION LOSSES AT THE THREE FREQUENCIES OF INTEREST

\begin{tabular}{|c||c|c|c|}
\hline Port number & $f_{1}$ & $f_{2}$ & $f_{3}$ \\
\hline \hline 1 & $6.7 \pm 0.4 \mathrm{~dB}$ & $7.0 \pm 0.3 \mathrm{~dB}$ & $7.0 \pm 0.5 \mathrm{~dB}$ \\
\hline 2 & $6.4 \pm 0.5 \mathrm{~dB}$ & $6.9 \pm 0.4 \mathrm{~dB}$ & $6.9 \pm 0.4 \mathrm{~dB}$ \\
\hline
\end{tabular}


TABLE V

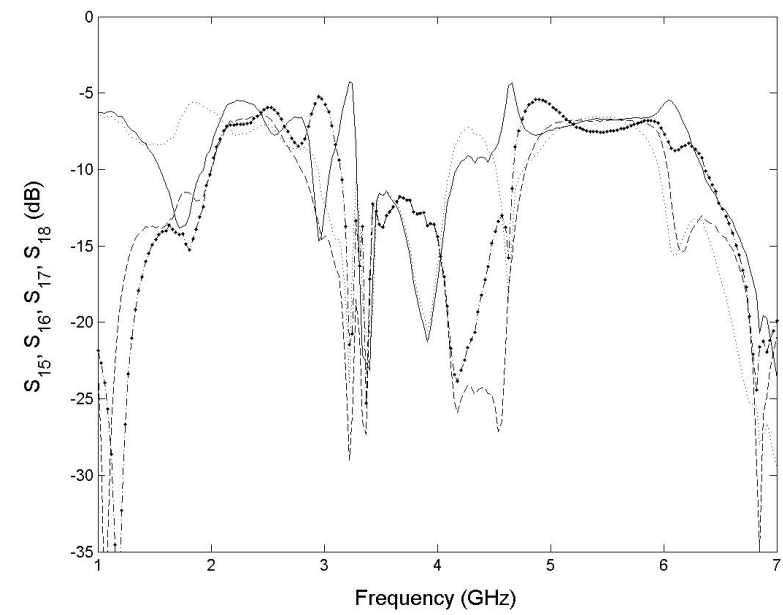

Fig. 8. Measurements of the insertion loss for the 1-5 (solid line), 1-6 (dashed line), 1-7 (dotted line) and 1-8 (dash-dot line) paths.

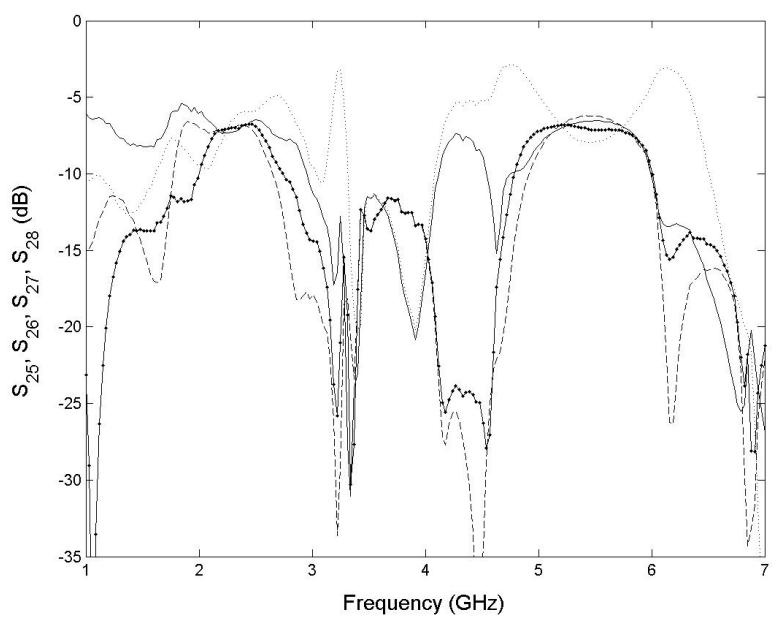

Fig. 9. Measurements of the insertion loss for the 2-5 (solid line), 2-6 (dashed line), 2-7 (dotted line) and 2-8 (dash-dot line) paths.

$\mathrm{BM}$ exhibits phase errors and couplings of within $13.5^{\circ}$ and $-6.7 \pm 0.8 \mathrm{~dB}$, respectively.

An ideal design of the proposed BM should create four radiation beams, two of them pointing at $\pm 14.5^{\circ}$ (boresight angle) for a progressive phase between antenna ports of $\pm 45^{\circ}$, and the other two pointing at $\pm 48.75^{\circ}$ for a progressive phase between antenna ports of $\pm 135^{\circ}$. An array of four isotropic antennas separated $0.5 \lambda_{0}$, being $\lambda_{0}$ the wavelength in the air, and with perfectly matched antenna ports to the BM network was considered. A side lobe level (SLL) of $11.3 \mathrm{~dB}$ should be expected in that case. Comparing the theoretical case with the measurements of the fabricated BM, errors in the scanning angles were predict to be approximately $1^{\circ}$ and $1.25^{\circ}$ for progressive phases of $\pm 45^{\circ}$ and $\pm 135^{\circ}$ respectively. The SLL was observed to be within $11.3 \mathrm{~dB}$ and $9.4 \mathrm{~dB}$.
PROGRESSIVE PHASE BETWEEN ANTENNA PORTS

\begin{tabular}{|c||c|c|c|}
\hline $\begin{array}{c}\text { Port } \\
\text { number }\end{array}$ & $f_{1}$ & $f_{2}$ & $f_{3}$ \\
\hline \hline 1 & $44.6^{\circ} \pm 7.4^{\circ}$ & $135.1^{\circ} \pm 8.7^{\circ}$ & $137.5^{\circ} \pm 9.9^{\circ}$ \\
\hline 2 & $-132.1^{\circ} \pm 7.1^{\circ}$ & $-42.7^{\circ} \pm 11.2^{\circ}$ & $-44.0^{\circ} \pm 10.3^{\circ}$ \\
\hline
\end{tabular}

\section{ACKNOWLEDGMENT}

Carlos Collado wants to thank the Ministry of Universities Research and Information Society (DURSI) of the Generalitat de Catalunya for hosting him at the Electrical Engineering and Computer Science Department at the University of California, Irvine. The authors also want to thanks the support of the Balsells fellowships and the California-Catalonia Engineering Innovation Program 2004.

\section{REFERENCES}

[1] M. Lisi, "An introduction to multiport power amplifiers," Microwave Engineering Europe, 1992.

[2] L. Accatino, A. Angelucci, and B. Piovani, "Design of butler matrices for multiport amplifier applications," Microwave Engineering Europe, pp. 45-50, Apr 1992.

[3] T. Denidni and T. Libar, "Wide band four-port butler matrix for switched multibeam antenna arrays," Personal, Indoor and Mobile Radio Coттиnications, vol. 3, Sept 2003.

[4] J. Butler and R. Lowe, "Beam forming matrix simplifies design of electronically scanned antennas," Applied Superconductivity, IEEE Transactions on, vol. 9, pp. 170-173, Apr 1961.

[5] H. Moody, "The systematic design of the butler matrix," Antennas and Propagation, IEEE Transactions on, vol. 12, no. 6, pp. 786-788, Nov 1964.

[6] C. Collado, A. Grau, and F. D. Flaviis, "A dual-band planar quadrature hybrid with enhanced bandwidth," Microwave Theory and Techniques, IEEE Transactions on, 2004, submitted.

[7] "http://www.agilent.com," Nov 2004.

[8] A. Corona and M. J. Lancaster, "A high-temperature superconducting butler matrix," Applied Superconductivity, IEEE Transactions on, vol. 13, no. 4, pp. 3867-3872, Dec 2003.

[9] K.-K. Cheng and F.-L. Wong, "A novel approach to the design and implementation of dual-band compact planar $90^{\circ}$ branch-line coupler," Microwave Theory and Techniques, IEEE Transactions on, vol. 52, no. 11, pp. 2458-2463, Nov 2004.

[10] T. Hirota, A. Minakawa, and M. Muraguchi, "Reduced-size branchline and rat-race hybrids for uniplanar mmic's," Microwave Theory and Techniques, IEEE Transactions on, vol. 38, no. 3, pp. 270-275, Nov 1990. 